\title{
Prevalence of Multidrug-Resistant and Extended-spectrum Beta-lactamase Producing Bacterial Isolates from Infected Wounds of patients in Kathmandu Model Hospital.
}

\author{
Kabita Adhikari ${ }^{1 *}$, Shaila Basnyat ${ }^{1}$ and Basudha Shrestha ${ }^{2}$ \\ ${ }^{1}$ Central Department of Microbiology, Tribhuvan University, Nepal \\ ${ }^{2}$ Kathmandu Model Hospital, Kathmandu, Nepal \\ *Corresponding Author \\ kabitaadhikarinepal@gmail.com
}

\begin{abstract}
The wound is an injury to living tissues caused by a cut, puncture, bite, blow, or other impacts. An infection is caused when germs enter wounds. This study was designed to isolate and identify the causative agents of wound infections and their antibiotic susceptibility pattern. A total of 339 samples were collected from January to June 2016 from Kathmandu Model Hospital, Kathmandu. Samples were inoculated on the Blood Agar and MacConkey agar plates were incubated at $37{ }^{\circ} \mathrm{C}$ for 24 hours. After incubation, all isolates were identified by using gram stain and biochemical methods. Antibiotic susceptibility tests were performed on Mueller Hinton agar plate by Kirby Bauer Disk Diffusion Technique. During the study period, altogether, 339 specimens were collected and processed as per the standard microbiological protocol. The overall prevalence of wound infection was $56.9 \%$. Among 244 bacterial isolates, Escherichiacoli (24.2\%) was most predominant bacteria followed by Staphylococcus aureus (19.7\%), Coagulase-negative Staphylococcus (17.6\%), Klebsiella pneumoniae (10.7\%), Pseudomonas aeruginosa (8.6\%), Acinetobacter spp (5.7\%), Citrobacter freundii (4.9\%) Proteus mirabilis (3.3\%), Streptococcus viridans (2.0\%), Klebsiella oxytoca (0.8\%), Proteus vulgaris $(0.4 \%)$, Serratia marcescens $(0.4 \%)$, Enterobacter aerogens $(1.2 \%)$, Enterobacter faecalis $(0.4 \%)$. The most effective drug for Gram-negative bacteria and Gram-positive bacteria were amikacin and chloramphenicol, respectively. A total of Gram-negative bacteria, $77.55 \%$ were multidrug-resistant. The total Gram-negative bacteria most ESBL producers were E. coli (82.9\%). We found S. aureus 33.3\% of isolates were resistant to cefoxitin which indicates the increasing rate of Methicillin-resistant $S$. aureus (MRSA) in wound infection.
\end{abstract}

\section{Keywords}

E.coli, MRSA, S. aureus, wound, infections

\section{Introduction}

The wound infection is a breach in the skin, and exposure of the subcutaneous tissues providea suitable environment for microbial colonization and proliferation (Yakha et al., 2014). In wound infections, bacteria deposit and multiply in tissue an associated host reaction (Collier et al., 2004). The main reason of wound infection is the breach in the skin that let different cell types enter the wound that initiates an inflammatory response. Signs of redness, pain, swelling, and fever are characteristics of the inflammatory response in the wound (Shrestha et al., 2009).
Wound infections can be caused by different groups of bacteria, which including Gram-positive and Gram-negative. In Gram-positive bacteria $S$. aureus, coagulase-negative $S$. aureus, Enterococci and Gram-negative bacteria E. coli, P. aeruginosa $K$. peumoniae, $K$. oxytoca, Enterobacter, $P$. mirabilis, P. vulgaris, Acinetobacter etc and other Streptococci and Candida (Gupta et al., 2002; Eselbehie et al., 2013).

Methicillin-resistant $S$. aureusis now endemic in most United States hospitals and long-term care facilities. Centres for Disease Control and Prevention of $S$. aureus isolates that are resistant 
to methicillin has increased steadily in recent years (Mera et al., 2011). MRSA is a significant pathogen causing health-related problems in the world (Chan et al., 2014).

This study may help to select appropriate empirical antibiotic treatment and may help in minimizing the alarming trend of antibiotic resistance which would be helpful for the management of such infections in the respective hospital.

\section{Methodology}

This study was carried out by collecting wound swabs and pus samples from patients visiting Kathmandu Model Hospital, Kathmandu from January to June 2016. A total of 339 samples were cultured on Blood agar and Mac Conkeyagar media incubated at $37^{\circ} \mathrm{C}$ for 24 hours. Organisms were identified by a standard microbiological procedure including colony characters, Gram staining and biochemical reactions. The antibiotic sensitivity test of all isolates was performed by modified Kirby Bauer disc diffusion method on Mueller Hinton agar or Blood agar medium using antibiotic discs of Hi media. After this isolated $S$. aureus was screened for methicillin resistance using cefoxitin disc $(30 \mu \mathrm{g})$ as per standard guidelines provided by CLSI, the zone of inhibition $\leq 21 \mathrm{~mm}$ is considered a positive result for MRSA strain. The test inoculum was matched with the Mac Farland tube 0.5 standard. Turbidity was prepared and carpet culture of Muller Hinton Agar (Cheesbrough 2006).

Screening of the suspected ESBL strains was performed according to the guidelines for screening issued by CLSI. According to this guideline, MDR isolates were screened for possible ESBL production using ceftriaxone $(30 \mu \mathrm{g})$, ceftazidime $(30 \mu \mathrm{g})$ and cefotaxime $(30 \mu \mathrm{g})$. Isolates were cefotaxime $\leq 27 \mathrm{~mm}$, ceftazidime $\leq 22 \mathrm{~mm}$ and ceftriaxone $\leq 25 \mathrm{~mm}$ were the possible ESBL producers. Regardless of the screening results, all the third generation cephalosporins resistant bacterial isolates were subjected to phenotypic confirmatory test using combined

Table 1. The pattern of bacterial isolates in wound samples

\begin{tabular}{|c|c|c|c|c|c|c|}
\hline \multirow{3}{*}{ Types of organism } & \multicolumn{4}{|c|}{ Sample } & \multicolumn{2}{|c|}{ Total } \\
\hline & \multicolumn{2}{|c|}{ Wound swab } & \multicolumn{2}{|c|}{ Pus aspirates } & \multirow{2}{*}{ Number } & \multirow{2}{*}{$\%$} \\
\hline & Number & $\%$ & Number & $\%$ & & \\
\hline E. coli & 31 & 23.3 & 28 & 25.2 & 59 & 24.2 \\
\hline S. aureus & 22 & 16.5 & 26 & 23.4 & 48 & 19.7 \\
\hline CoNS & 24 & 18.0 & 19 & 17.1 & 43 & 17.6 \\
\hline K. pneumoniae & 15 & 11.3 & 11 & 9.9 & 26 & 10.7 \\
\hline P. aeruginosa & 14 & 10.5 & 7 & 6.3 & 21 & 8.6 \\
\hline Acinetobacterspp & 7 & 5.3 & 7 & 6.3 & 14 & 5.7 \\
\hline C.freundii & 9 & 6.8 & 3 & 2.7 & 12 & 4.9 \\
\hline P. mirabilis & 6 & 4.5 & 2 & 1.8 & 8 & 3.3 \\
\hline S.viridans & 1 & 0.8 & 4 & 3.6 & 5 & 2.0 \\
\hline K. oxytoca & 1 & 0.8 & 1 & 0.9 & 2 & 0.8 \\
\hline P. vulgaris & 1 & 0.8 & 0 & 0.0 & 1 & 0.4 \\
\hline S. marcescens & 0 & 0.0 & 1 & 0.9 & 1 & 0.4 \\
\hline E. aerogens & 2 & 1.5 & 1 & 0.9 & 3 & 1.2 \\
\hline E. faecalis & 0 & 0.0 & 1 & 0.9 & 1 & 0.4 \\
\hline Total & 133 & 100 & 111 & 100 & 244 & 100 \\
\hline
\end{tabular}


Nepal Journal of Science and Technology (NJST) (2020), 19(1)

Table 2. Antibiotic susceptibility pattern of $E$. coli

\begin{tabular}{lcccccc}
\hline & \multicolumn{6}{c}{ Susceptibility pattern } \\
\cline { 2 - 7 } Antibiotics & \multicolumn{2}{c}{ Sensitive } & \multicolumn{2}{c}{ Intermediate } & \multicolumn{2}{c}{ Resistant } \\
\cline { 2 - 7 } & No & $\%$ & No & $\%$ & No & $\%$ \\
\hline Amikacin & 49 & 83.1 & - & - & 10 & 81.4 \\
Amoxycillin & 11 & 18.6 & - & - & 47 & 81.4 \\
Amoxyclav & 11 & 18.6 & - & - & 47 & 69.5 \\
Ceftazime & 18 & 30.5 & - & - & 41 & 69.5 \\
Ceftriaxone & 18 & 30.5 & - & - & 41 & 8.5 \\
Chloramphenicol & 54 & 91.5 & - & - & 5 & - \\
Colistin & 59 & 100 & - & - & - & 66.1 \\
Co-trimoxazole & 20 & 33.9 & - & - & 39 & 15.3 \\
Doxycycline- & 49 & 83.1 & 1 & 1.7 & 9 & 23.7 \\
Hydrochloride & & 71.2 & 3 & 5.1 & 14 & 6.8 \\
Gentamycin & 42 & 91.5 & 1 & 1.7 & 4 & 55.9 \\
Imipenem & 54 & 37.3 & 4 & 6.8 & 33 & 6.8 \\
Levofloxacin & 22 & 91.5 & 1 & 1.7 & 4 & 55.9 \\
Meropenem & 54 & 37.3 & 4 & 6.8 & 33 & 6.8 \\
Ofloxacin & 22 & 91.5 & 1 & 1.7 & 4 & - \\
Piperacillin/ & 54 & 100 & - & - & - & - \\
Tazobactam & 59 & 100 & - & - & - & \\
Polymixin-B & 59 & & & & \\
Tigecycline & 54 & & & & \\
\hline
\end{tabular}

Table 3. Antibiotic susceptibility pattern of $S$. aureus

\begin{tabular}{|c|c|c|c|c|c|c|}
\hline \multirow{3}{*}{ Antibiotics } & \multicolumn{6}{|c|}{ Susceptibility pattern } \\
\hline & \multicolumn{2}{|l|}{ Sensitive } & \multicolumn{2}{|c|}{ Intermediate } & \multicolumn{2}{|l|}{ Resistant } \\
\hline & No & $\%$ & No & $\%$ & No & $\%$ \\
\hline Amoxycillin & 3 & 6.2 & - & - & 45 & 93.8 \\
\hline Amoxyclav & 3 & 6.2 & - & - & 45 & 93.8 \\
\hline Cephalexin & 31 & 64.6 & - & - & 17 & 35.4 \\
\hline Chloramphenicol & 45 & 93.6 & - & - & 3 & 6.4 \\
\hline Ciprofloxacin & 20 & 40.4 & - & - & 28 & 59.6 \\
\hline Co-trimoxazole & 15 & 29.8 & - & - & 33 & 70.2 \\
\hline $\begin{array}{l}\text { Doxycycline- } \\
\text { Hydrochloride }\end{array}$ & 43 & 89.4 & 1 & 2.1 & 4 & 8.5 \\
\hline Erythromycin & 33 & 68.1 & - & - & 15 & 31.9 \\
\hline Gentamycin & 43 & 89.4 & - & - & 5 & 10.6 \\
\hline Teicoplanin & 48 & 100.0 & - & - & - & - \\
\hline Tigecycline & 48 & 100.0 & - & - & - & - \\
\hline Vancomycin & 48 & 100.0 & - & - & - & - \\
\hline
\end{tabular}


Table 4. Multidrug-resistant Gram-negative bacteria

\begin{tabular}{|c|c|c|c|c|}
\hline \multirow{3}{*}{ Bacteria } & \multicolumn{4}{|c|}{ MDR Bacteria } \\
\hline & \multicolumn{2}{|c|}{ MDR } & \multicolumn{2}{|c|}{ No MDR } \\
\hline & NO & $\%$ & No & $\%$ \\
\hline E. coli & 46 & 78.0 & 13 & 22.0 \\
\hline K. pneumoniae & 23 & 88.2 & 3 & 11.5 \\
\hline P. aeruginosa & 12 & 57.1 & 9 & 42.9 \\
\hline Acinetobacterspp & 13 & 92.9 & 1 & 7.1 \\
\hline P. mirabilis & 7 & 87.5 & 1 & 12.5 \\
\hline C. freundii & 8 & 66.7 & 4 & 33.3 \\
\hline K oxytoca & 2 & 100.0 & - & - \\
\hline P. vulgaris & 1 & 100.0 & - & - \\
\hline S. marcescens & - & - & 1 & 100 \\
\hline E. aerogens & 2 & 66.7 & 1 & 33.3 \\
\hline Total & 114 & & 33 & \\
\hline
\end{tabular}

disks test (CDT). ESBLs set consisting of Set 1: ceftazidime $(30 \mu \mathrm{g})$ and ceftazidime $(30 \mu \mathrm{g})$ plus clavulanic acid $(10 \mu \mathrm{g})$, Set 2 : cefotaxime $(30 \mu \mathrm{g})$ and cefotaxime $(30 \mu \mathrm{g})$ plus clavulanic acid (10 $\mu \mathrm{g})$. The zone of inhibition for the ceftazidime and cefotaxime discs were compared to that of the ceftazidime and cefotaxime plus clavulanic acid combination discs. An increase in the zone diameter of $\geq 5 \mathrm{~mm}$ in the presence of clavulanic acid, from any or all of the kit sets, was concluded as confirmed ESBL producers.

\section{Results and Discussion}

From a total of 339 wound samples, 193 (56.9\%) samples showed aerobic bacterial growth and $43.1 \%$ were growth negative (figure 1 ).

Out of a total of 168 wound swab, $102(52.8 \%)$ were positive and also out of 171 pus aspirates 91 (47.2\%) were positive. In this study, 187 (58.1\%) samples from male patients and among them, 112 (56.9\%) were positive. 142 (41.9\%) samples were from female patients, and among them, 81 (57\%) were positive. Out of 193 positives samples, 146 (75.6\%) showed monomicrobial growth, and 47 (24.4\%) showed polymicrobial growth.

Out of 244 bacterial isolates obtained from the 193 positive samples, 97 (39.8\%) bacterial isolates were Gram-positive, and 147 (60.2\%) bacterial isolates were Gram-negative. The most common bacterial isolates were $E$. coli, followed by $S$. aureus. Among Gram-positive S.aureus 48 $(19.7 \%)$ were most common isolates followed by CoNS $43(17.6 \%), S$. viridans $5(2.0 \%)$ and E. faecalis $1(0.4 \%)$.From a total of $48 \mathrm{~S}$. aureus were isolated from wound samples, 16 (33.3\%) were MRSA. Among total positive isolates, 147 were Gram-negative, of which 59 (24.2\%) were the most common isolates followed by $K$. pneumoniae 26 (10.7\%). P. aeruginosa 21 (8.6\%), Acinetobacterspp14 (5.7\%), C. freundii 12 (4.9\%), P. mirabilis $8(3.3 \%)$, K. oxytoca $2(0.8 \%), P$. vulgaris $1(0.4 \%), S$. marcescens $1(0.4 \%)$ and $E$. aerogens $3(1.2 \%)$ (Table 1$)$.

The most susceptible first-line antibiotic for E.coli $(\mathrm{n}=59)$ was amikacin. Among the total isolated E. coli, $83.1 \%$ were susceptible to amikacin and levofloxacin. Ofloxacin was the second most effective antibiotic against the $37.3 \%$ E. coli. Similarly, $18.6 \%$ of $E$. coli were least susceptible to amoxycillin and amoxyclav. Among the isolated E. coli, $91.5 \%$ were susceptible to meropenem, imipenem, piperacillin/tazobactam, and chloramphenicol and $100 \%$ of isolated E. coli were susceptible to third-line antibiotics colistin, polymixin-B, and tigecycline (Table 2 ).

The most effective antibiotic for $S$. aureus $(n=48)$ was chloramphenicol $(93.6 \%)$ followed by gentamycin and doxycycline (89.4\%). The least susceptible to amoxycillin and amoxyclav 
(6.2\%). Among the isolated S. aureus $100 \%$ were susceptible to second-line antibiotics vancomycin, teicoplanin, and tigecycline (Table 3).

Among the 147 Gram-negative bacteria, 114 were multidrug-resistant, and 33 were nonmultidrug resistant. The isolated MDR bacteria were E. coli 46 (78\%), K. pneumonia 23 (88.2\%), P. aeruginosa 12(57.1\%), Acinetobacterspp $13(92.9 \%)$, P. mirabilis 7 (87.5\%),C. freundii $8(66.7 \%), K$. oxytoca $2(100 \%)$, P.vulgaris 1 $(100 \%)$ and E. aerogens $3(66.7 \%)$ (Table 4).

A total of 41 isolates of MDR E. coli, 34 isolates were ESBL producers. Among 20 isolates of MDR $K$. pneumoniae, 10 isolates were ESBL producers. Similarly, out of 12 isolates ofMDR Acinetobacterspp, 2 isolates were ESBL producers. Also, 12 isolates were MDR $P$. aeruginosa, 1 isolate was ESBL producers, a total of 8 isolates of MDR $C$. freundii, 5 isolates were ESBL producers, among 3 isolates of MDR P. mirabilis, 2isolates were ESBL producers. Besides, a total of 2 isolates of MDR K. oxytoca, 1 isolate was ESBL producers, but no isolates of $P$. vulgaris and E. aerogens were ESBL producers (Table 5).

Table 5. ESBL producers among MDR Gram negative bacteria

\begin{tabular}{lccc}
\hline \multirow{2}{*}{ Bacterial isolates } & Total & \multicolumn{2}{c}{ ESBL } \\
& & No & $\%$ \\
\hline E. coli & 41 & 34 & 82.9 \\
K. pneumoniae & 20 & 10 & 50.0 \\
P. aeruginosa & 12 & 1 & 8.3 \\
Acinetobacterspp & 12 & 2 & 16.7 \\
C. freundii & 8 & 5 & 62.5 \\
P. mirabilis & 3 & 2 & 66.7 \\
K. oxytoca & 2 & 1 & 50.0 \\
P. vulgaris & 1 & 0 & 0.0 \\
E. aerogens & 2 & 0 & 0.0 \\
\hline
\end{tabular}

In this study, out of 339 samples collected, 193 (56.9\%) samples showed aerobic bacterial growth, and $146(43.1 \%)$ samples showed no growth. The overall prevalence rate of wound infections was 50\% (Shrestha et al.,2009). In a similar study conducted at TUTH, $50.7 \%$ of total samples showed growth (Acharya et al.,2008) and 49.3\% with no growth. Similarly, a study carried out by Chitwan Medical College Teaching Hospital showed that out of 200 samples $150(75 \%)$ showed growth (Gautam et al., 2013) and 60\% showed the growth positive (Bhatta and Lakhey 2007).

In a present study, out of total samples collected, 187 (58.1\% were collected from male patients, and $112(41.9 \%)$ were collected from female patients. Though our study showed a higher number of male cases than female cases, the growth rate didn't differ significantly between male and female populations ( $p>0.05)$. A similar study was carried out in Lahore which showed $20 \%$ more male patients than females (Zafar et al., $2007)$ and a higher percentage of males (76.5\%) patients were found than females in Nigeria (Adegoke et al., 2010).

Out of 193 positives samples, 146 (75.6\%) showed monomicrobial Growth, and 47 (24.4\%) showed polymicrobial growth. The single isolate was higher than multiple isolates in both pus swab and aspirated pus. Various studies carried out in wound infection showed a higher rate of monomicrobial infection than polymicrobial infection (Karki 2012; Komolafe et al., 2003; Kumari 2008; Nepal and Shrestha et al., 2009).

We identified 244 bacterial isolates obtained from the 193 positive samples, 97 (39.8\%) bacterial isolates were Gram-positive, and 147 (60.2\%) bacterial isolates were Gram-negative. Another study from Kathmandu Model hospital showed that among the total isolates, $273(64.08 \%)$ were Gram-positive bacteria, and 153 (35.92\%) were Gram-negative bacteria (Shrestha et al., 2009). In wound swab, E. coli $31(23.3 \%)$ was most predominant, followed by 24 (18\%) CoNS and $S$. aureus $22(16.5 \%)$. Similarly, in pus aspirates $E$. coli $28(25.2 \%)$ was most predominant, followed by S. aureus 26 (23.4\%) and CoNS 19 (17.1\%).

We found that the most common bacterial isolates were E. coli, followed by $S$. aureus. The most predominant bacteria were E. coli supported by (Gautam et al., 2013; KC et al., 2013). The most predominance of $S$. aureus and the second most predominant bacteria is $E$. coli in a wound is supported by many studies (Karki 2012; Shrestha 
et al.,2009). The predominance of E. coli in a wound is supported by many studies (Gautam et al., 2013). Among the 244 bacterial isolates, 14 different species were isolated. (Kansakar et al., 2003 ) in TUTH, which reported that $82.5 \%$ of the sample cultured aerobically showed bacterial growth and 13 different bacterial species were isolated. S. aureus was the most frequently isolated organisms (57.7\%), followed by E. coli $(11 \%)$ and CoNS (3\%) (Basnet 2011), found that the most predominant organism was S. aureus (19.71\%) followed by E. coli (15.5\%). Gautam et al., (2013), found most predominant bacterial species as $S$. aureus $(65.3 \%)$ followed by $K$. pneumoniae (8\%), E. coli (7.3\%), CoNS (6\%), P.aeruginosa (5.3\%), Enterococcus spp., (3.3\%), Enterobacter spp., (2\%), Acinetobacter spp. (1.3\%), P. mirabilis $(0.6 \%)$ and P. vulgaris $(0.6 \%)$. S.aureuswas the predominant organism followed by hemolytic Streptococcus (Ruth and Keith 2004). Bhatta and Lakhey (2007); Singh et al., (2006) and Shrestha et al., (2009) reported that after S.aureus, E. coli was the second predominant isolate. Mumtaz et al., (2002), samples from aerobic pyogenic isolates from wounds and abscesses, reported that $S$. aureus was the most common pathogen (49\%) followed by E. coli $(25.9 \%)$, Klebsiella (9.5\%), P. aeruginosa (8.6\%), Proteus spp. (4\%) and Acinetobacterspp (2.7\%). Another study conducted by B.D. Sharma Postgraduate Institute of Medical Sciences in Rohtak, India found that the most common wound isolate was $S$. aureus (32.3\%) followed by K. pneumoniae $(22.0 \%)$, Pseudomonas spp (18.7\%) and E.coli(17.4\%) (Gupta et al., 2002).

This study also showed that the most susceptible first-line antibiotic was amikacin and among the total isolated E. coli $83.1 \%$ were susceptible to amikacin, levofloxacin, and ofloxacin were second most effective antibiotic against the $37.3 \%$ E. coli. Similarly, $18.6 \%$ E. coli were least susceptible to amoxycillin and amoxyclav. Among the isolated E. coli, $91.5 \%$ were susceptible to meropenem,imipenem,piperacillin/ tazobactam, and chloramphenicol and $100 \%$ of isolated $E$. coli were susceptible to third-line antibiotics colistin, polymixin-B, and tigecycline. E. coli was found to be sensitive to gentamycin $(80 \%)$, ciprofloxacin $(60 \%)$, cefotaxime $(50 \%)$ and co-trimoxazole (40\%). The least effective antibiotic was ampicillin followed by cephazolin $(20 \%)$ and ceftriaxone $(30 \%)$. A study conducted by Karkee (2008) in Bir Hospital, 78\% of isolates were sensitive to gentamycin whereas $55.3 \%$ of isolates were resistant to ciprofloxacin, $65.8 \%$ were equally resistant to co-trimoxazole and amoxicillin. In a study carried out by Nwachukwa et al., (2009), 55\% of E. coli isolates were sensitive to ciprofloxacin. The studies carried out by Bhatta and Lakhey (2007), and Singh et al., (2006) found that $E$. coli was equally susceptible to cephalexin, co-trimoxazole, and ciprofloxacin (57\%).

The most effective antibiotic in firstline antibiotics was chloramphenicol (93.6\%) followed by gentamycin and doxycycline (89.4\%). The least susceptible to amoxycillin and amoxyclav (6.2\%). Among the isolated S. aureus $100 \%$ were susceptible to second-line antibiotics vancomycin, teicoplanin, and tigecycline. Gautam et al., (2013) have found that $S$. aureus was highly sensitive to amikacin (83.6\%) followed by ceftriaxone $(67.3 \%)$, ciprofloxacin $(65.3 \%)$, cefotaxime $(55 \%)$, gentamycin $(53.06 \%)$. It was highly resistant to ampicillin (67.3\%), and cotrimoxazole (65.3\%) Andragachew et al., (2006) has reported ampicillin (55\%) and co-trimoxazole $(65 \%)$ as a highly resistant drug against S.aureus.

Similarly, out of 147 Gram-negative bacteria, 114 were multidrug-resistant, and 33 were nonmultidrug-resistant. The isolated MDR bacteria were E. coli 46 (78\%), K. pneumoniae 23 (88.2\%), P. aeruginosa 12 (57.1\%), Acinetobacter spp 13 (92.9\%), P. mirabilis 7 (87.5\%), C. freundii 8 (66.7\%), K. oxytoca 2 (100\%), P.vulgaris $1(100 \%)$ and E. aerogens $3(66.7 \%)$. A similar study found in (Edward et al., 2013), A. baumannii isolates recovered from patients with burns greater than $30 \%$ of total body surface were more likely to be MDR (61\%) with no significant difference for $P$. aeruginosa and $K$. pneumoniae. Another study found that total $P$. aeruginosa isolates, 62 were found to multidrug resistance, of which 2 were resistant to three antimicrobial classes. (Yakha et al., 2014). In a similar study, overall multi-drug resistant isolates were $66.7 \%$ (Raza et al., 2013).

From the total 48 (23.4\%) S. aureus isolated 
from wound samples, $16(33.3 \%)$ were MRSA. The overall prevalence of MRSA was $68 \%$ (Khanal et al., 2010). Out of 36 S. aureus, 15 isolates were MRSA (Raza et al., 2013).

Out of 41 isolates of MDR E. coli, 34 isolates were ESBL producers. Likewise, among 20 isolates of MDR K. pneumoniae, 10 isolates were ESBL producers. Similarly, a total of 12 isolates of MDR Acinetobacter spp, 2 isolates were ESBL producers. Also, 12 isolates MDR P. aeruginosa, 1 isolate was ESBL producers, a total of 8 isolates of MDR C. freundii and 5 isolates were ESBL producers. Besides, a total of 3 isolates of MDR $P$. mirabilis, 2 isolates were ESBL producers, among 2 isolates of MDR $K$. oxytoca, 1 isolate was ESBL producers but no isolates of $P$. vulgaris and $E$. aerogens were ESBL producers. In the tertiary care hospital of eastern Nepal, A total of 300 Gram-negative bacilli isolated from the pus samples were identified phenotypically, and antimicrobial activity was determined. MDR was found in $92.6 \%$ of ESBL producers (Shrestha et al., 2011).

Another study in pus and wound swabs from Saudi Arabia, E. coli (21) and K. pneumoniae (11) were found to be ESBL producers. The highest numbers of ESBL producing E. coli were detected by cefpodoxime, followed by aztreonam, ceftazidime, cefotaxime, and ceftriaxone. For ESBL producing K. pneumoniae, it was cefpodoxime followed by cefotaxime, ceftazidime, aztreonam, and ceftriaxone (AlZahrani et al., 2005).

In a study from Uganda, the ESBL producing Gram-negative bacteria in wound swab was $100 \%$, and in pus swab was $47.4 \%$ (Kateregg et al., 2015). In Saudi Arabia, among the $K$. pneumoniae isolated from pus sample, $50 \%$ were found to be ESBL producers. This study was done by the double-disk synergy test method (Rahim et al., 2014).

Among Enterobacteriaceae isolates, 25\% of isolates of E. coli were ESBL producers, $40 \%$ of K. pneumoniaeisolates were ESBL producer and $33.3 \%$ of $C$. freundii were ESBL producer. But no species of Proteus were ESBL producers. Baral
(2008) showed the presence of $28.12 \%$ ESBL producers out of 96 MDR isolates, Bomjan (2005) found the presence of $28.3 \%$ ESB producers among various clinical isolates and Sharma (2004) found $8 \%$ K. pneumoniae, $12.5 \%$ E. coli, $12.5 \%$ C.freundii, $25 \%$ A.calcoaceticus and $5 \% P$. aeruginosa as ESBL-producing strains. Poudyal (2010) reported $62.72 \%$ of ESBL producers, of which $86.96 \%$ were E. coli. Of all the organisms studied till date, the most potent ESBL producers belong to the family Enterobacteriaceae (E. coli, K. pneumoniae, E.aerogenes, P. mirabilis, etc. (Bradford 2001; Senekal 2010).

Wound infections have a problem in the field of medicine for a long time. Advances in control of infections have not completely eradicated this problem because of the emergence of drug resistance (Thomas 1991). As compared to the previous study done, antibiotic resistance pattern is increasing. Many factors may have contributed to such a level of resistance, including the misuse of antibiotics by health professionals and unskilled practitioners (Karki 2012). In Nepal, it is a common practice that antibiotics can be purchased without a prescription, which leads to the misuse of antibiotics, thus contributing to the emergence and spread of antimicrobial resistance. MRSA is proving to be the scourge of modern-day surgery and can colonize the skin and body like other strains; they appear to be increasing in frequency and are displacing resistance to a broader range of antibiotics including vancomycin. Hence, they must be considered a severe problem.

\section{Conclusion}

Gram-negative bacteria were found to be more predominant compared to Gram-positive bacteria in wound infections. E. coli was one of the major pathogens responsible for causing wound infections followed by $S$. aureus. Besides these, other organisms most frequently encountered in this study were $P$. aeruginosa, Acinetobacter spp, Enterobacter spp, C. freundii, K. pneumoniae, CoNS, P. vulgaris, P. mirabilis, S. viridans, $E$. faecalis, $S$. marcescens, and $K$. oxytoca.

Gentamycin and chloramphenicol were the most effective for both Gram-positive and Gram- 
negative organisms. Most of the organisms were resistant to amoxycillin, ceftazidime, and cotrimoxazole. Among total isolates of $S$. aureus, $33.3 \%$ of isolates were found to be resistant to cefoxitin which indicated the increasing rate of MRSA in wound infection. Among total Gramnegative bacteria, $82.9 \% \mathrm{E}$. coli were ESBL producers followed by $P$. mirabilis $66.7 \%$. Increasing the resistance pattern of antibiotics is being a threat to human life which is progressing at an alarming rate.

\section{Acknowledgements}

We acknowledge the Master Thesis support grant provided by the University Grants Commission (UGC). We are very thankful to Kathmandu Model Hospital, Kathmandu and Central Department of Microbiology, Tribhuvan University, Kritipur, Nepal for making facilities available.

\section{References}

Adegoke, A.A. and A.O. Komolafe.2008. Nasal colonization of school children in Ile-Ife by multiple antibiotic-resistant Staphylococcus aureus.Int J Biotech All Sci 3: 3876-3881.

Al-kasaby, N. and V. Sachdeve. 2015. Antibiotic resistance patterns of bacterial isolates in the adult intensive care unit at Nizwa Hospital, Oman. British microbiology journal 10: 1-10.

Banjara, M.R., A.P. Sharma, A.B. Joshi, N.R. Tuladhar, P. Ghimire and D.R. Bhatta. 2003. Surgical wound infection in Tribhuvan University Teaching Hospital. Journal of Nepal Health Research Council 1: 41-45.

Bhatta, C.P. and M. Lakhey. 2007. The distribution of pathogens causing wound infection and their antibiotic susceptibility pattern. J NEP Health Res Counc 5: 22-25.

Chan, W.S., B.S. Tang, M.V. Boost, C. Chow, and P.H. Leucing. 2014. Detection of methicillin-resistant Staphylococcus aureus using a gold nanoparticlebased colourimetric polymerase chain reaction assay. Biosensors and bioelectronics 53: 105-111.

Cheesbrough M (2006). District Laboratory Practice in Tropical Countries. 2nd edn. Cambridge. Newyork Melbourne, Madrid, Cape Town, Singapore, Sao Poulo: Cambridge University Press.

Church, D., S. Elsayed, O. Reid, B. Winston and R. Lindsay. 2006. Burn wound infection. Clin. Microbiol. Rev. 19: 403-434.CLSI. 2013. Performance standards for antimicrobial susceptibility testing. Twenty-Third InformationalSupplement. 33:1-199.

Collier, M., 2002. Wound bed management, key principles for practice. Prof. Nurs 18: 221-225

Esebelahie, N.O., F.O. Esebelahieand R. Omoregie. 2013. Aerobic bacterial isolates from wound infection.Afr. J. Cln. Exper. Microbiol. 14: 155159.

Gautam, R., A. Acharya, H.P. Nepal and S. Shrestha. 2013. Antibiotic susceptibility pattern of bacterial isolates from wound infection in Chitwan medical college teaching hospital, Chitwan Nepal. International journal of biomedical and advance research 4: 249-252.

Gupta, N., V. Gautam, S. Saini, L. Singh, and D.R. Arora. 2002. Prevalence of multidrug organism in wound infection. J. Infect. Dis. Antimicrobial. Agent. 19: 111-117.

Karki, S., 2012. Antibiotic susceptibility pattern of bacterial isolates from wound infection in patients visiting Kanti Children's Hospital. M.Sc. Dissertation submitted to the Central Department of Microbiology, Tribhuvan University.

Kateregga, J.N., R. Kantume, C. Atuhaire, M.N. Lubowaand J. Ndukui.2015. Phenotypic expression and prevalence of ESBL- producing Enterobacteriaceae in samples collected from patients in various wards of Mugalo Hospital Uganda. BMC pharmacology and toxicology 16:14.

KC, R., A. Shrestha and V.K. Sharma. 2013. Bacteriological study of wound infection and antibiotic susceptibility pattern of the isolates. Nepal journal of science and technology 14: 143150.

Khanal, L.K. and B.K. Jha. 2010. Prevalence of methicillin-resistant Staphylococcus aureus (MRSA) among skin infection cases at a hospital in Chitwan, Nepal. Nepal med coll J 12: 224-228.

Komolafe, O., J. James, L. Dalongolera and M. Koka. 2003. Bacteriology of burns at the Queen Elizabeth Central Hospital, Blantyre, Malawi. Burns 29: 235-238

Kumari, K. 2008. A pattern of bacterial isolates and antibiogram from open wound infection among the indoor patients of Bir Hospital. M.Sc. Dissertation submitted to the Central Department of Microbiology, Tribhuvan University.

Mera, M.A, Abdissa and T. Sewunet. 2014. Antimicrobial susceptibility pattern of bacterial isolates from wound infection and their sensitivity to alternative topical agents at Jimma University specialized hospital, south-west Ethiopia. Annals of clinical microbiology and antimicrobials 13: 14 . Rakhem, K.A.A. and A.M.A. Mohamed. 2014. 
Nepal Journal of Science and Technology (NJST) (2020), 19(1)

Prevalence of extended-spectrum beta-lactamaseK. pneumonia in clinical isolates. Jundispur $J$ Microbial 7: e17114

Rani, R.V. and J. Nithyalakshmi. 2014. A comparative study of diabetic and non-diabetic wound infections with special reference to MRSA and ESBL. International current Microbiol applied sciences 3: 546-554.

Raza, M.S., A. Chander, A. Ranabhat. 2013. Antimicrobial susceptibility patterns of the bacterial isolates in post-operative wound infections in a tertiary care Hospital, Kathmandu, Nepal. Open journal of medical microbiology 3:159-163.

Shrestha, B. and R.B. Basnet. 2009. Wound infection and antibiotic sensitivity pattern of bacterial isolates. PMJN9: 1-6.
Singh, A.P. Vaidya, N.R. Tuladharand S. Maharjan. 2006. Drug sensitivity pattern of microorganisms in infected wounds at TUTH. Journal of the Institute of medicine 28: 55-56.

Upadhyay, A.K., R. Maharjan and B. Shakya. 2012. Multidrug resistance bacteria in different clinical samples in National Medical College and Teaching Hospital, Birgunj, Nepal. RJPBC S 3: 797-807.

Yakha, J.K., A.R.Sharma, N. Dahal, B. Lekhak and M.R. Banjara. 2015. Antibiotic susceptibility pattern of bacterial isolates, causing wound infection among the patients visiting B\&B Hospital. Nepal journal of science and technology 15: 91-96.

Zafar, A., N. Anwar and H. Ejaz. 2008. Bacteriology of infected wounds- a study conducted at children hospital Lahore. E/Biomedica 23: 8. 